\title{
Testing CPT Invariance with Neutrinos
}

\author{
Tommy Ohlsson ${ }^{\mathrm{a} * \dagger}$ \\ aDivision of Mathematical Physics, Department of Physics, Royal Institute of Technology (KTH) - \\ Stockholm Center for Physics, Astronomy, and Biotechnology (SCFAB), \\ Roslagstullsbacken 11, 10691 Stockholm, Sweden
}

\begin{abstract}
We investigate possible tests of CPT invariance on the level of event rates at neutrino factories. We do not assume any specific model, but phenomenological differences in the neutrino-antineutrino masses and mixing angles in a Lorentz invariance preserving context, which could be induced by physics beyond the Standard Model. We especially focus on the muon neutrino and antineutrino disappearance channels in order to obtain constraints on the neutrino-antineutrino mass and mixing angle differences. In a typical neutrino factory setup simulation, we find, for example, that $\left|m_{3}-\bar{m}_{3}\right| \lesssim 1.9 \cdot 10^{-4} \mathrm{eV}$ and $\left|\theta_{23}-\bar{\theta}_{23}\right| \lesssim 2^{\circ}$.
\end{abstract}

\section{INTRODUCTION}

The theme of the current presentation is to discuss the plausibility of testing possible CPT invariance violation in the neutrino sector using future so-called neutrino factories.

The CPT theorem is one of the milestones of local quantum field theory (QFT). Moreover, the standard model (SM) of elementary particle physics is in very good agreement with all (at least most) existing experimental data. The CPT theorem is valid for the SM. Thus, CPT violation is connected with the search for physics beyond the SM. So far, no CPT violation has been found. However, neutrinos have been suggested as a source of CPT violation.

Prior papers on CPT violation with neutrinos include Refs. [1, 2, 3, An. Recently, neutrinos as a source of CPT violation have been investigated by several authors [5,6, 7, 8, 9, 10, 11, 12, 13, 14,. Some of the models of CPT violation with neutrinos (beyond the SM) are the following:

- S.R. Coleman and S.L. Glashow [1,2]: A Lorentz and CPT-violating model is introduced in which the most general CPT-

\footnotetext{
*E-mail: tommy@theophys.kth.se

$\dagger$ In collaboration with: S.M. Bilenky, M. Freund, M. Lindner, and W. Winter. Talk presented at the XXXIst International Conference on High Energy Physics (ICHEP 2002), Amsterdam, The Netherlands, July 24-31, 2002.
}

violating interaction $u^{\dagger} b u$ is allowed, where $b$ is a Hermitian matrix. This interaction implies that the energies of the ultrarelativistic neutrinos with definite momentum $p$ are the eigenvalues of the matrix:

$$
c p+\frac{m^{2}}{2 p}+b,
$$

where $m^{2} \equiv m m^{\dagger}$ is the Hermitian mass squared matrix ( $m$ is a complex symmetric mass matrix) and $c$ is also a Hermitian matrix, which describes velocity-mixing. Limiting the discussion to neutrino oscillations with two flavors, the neutrino oscillation transition probability formula becomes

$$
\begin{gathered}
\mathrm{P}\left(\nu_{\alpha} \rightarrow \nu_{\alpha^{\prime}}\right)=1-\sin ^{2} 2 \theta \\
\times \quad \sin ^{2}\left[\left(\frac{\Delta m^{2}}{4 E}+\frac{\Delta b}{2}+\frac{\Delta c E}{2}\right) L\right]
\end{gathered}
$$

where $\Delta m^{2}, \Delta b$, and $\Delta c$ are the differences between the eigenvalues of the matrices $m^{2}$, $b$, and $c$, respectively. Note that in order for the above formula to hold the mixing angles that diagonalize the matrices $m^{2}, b$, and $c$ need all to be equal to each other. 
- V.D. Barger et al. [3]: The effective Lorentz and CPT-violating interaction for neutrinos is: $\bar{\nu}_{L}^{\alpha} b_{\alpha \alpha^{\prime}}^{\mu} \gamma_{\mu} \nu_{L}^{\alpha^{\prime}}$, which implies that

$$
\begin{aligned}
\Delta \mathrm{P}_{\alpha \alpha}^{\mathrm{CPT}} & \equiv \mathrm{P}\left(\nu_{\alpha} \rightarrow \nu_{\alpha}\right)-\mathrm{P}\left(\bar{\nu}_{\alpha} \rightarrow \bar{\nu}_{\alpha}\right) \\
& =-2 \sin ^{2} 2 \theta \sin \left(\frac{\Delta m^{2} L}{2 E}\right) \\
& \times \sin (\Delta b L)
\end{aligned}
$$

when the matrices $m^{2}$ and $b$ are diagonalized by the same mixing angle $\theta$.

- G. Barenboim et al. [6]: CPT violation (but no Lorentz violation) is suggested to be so strong that the mass spectra of neutrinos and antineutrinos are completely different. This means that it would be possible to describe solar, atmospheric, and LSND neutrino data at the same time. Such a model is accomplished by changing the Hamiltonian from

$H_{0}=\int \frac{d^{3} p}{(2 \pi)^{3}}\left(\mathbf{p}^{2}+m^{2}\right) \sum_{s}\left[a_{\mathbf{p}}^{s \dagger} a_{\mathbf{p}}^{s}+b_{\mathbf{p}}^{s \dagger} b_{\mathbf{p}}^{s}\right]$

to

$$
\begin{aligned}
H_{0} & =\int \frac{d^{3} p}{(2 \pi)^{3}} \sum_{s}\left[\left(\mathbf{p}^{2}+m^{2}\right) a_{\mathbf{p}}^{s \dagger} a_{\mathbf{p}}^{s}\right. \\
& \left.+\left(\mathbf{p}^{2}+\bar{m}^{2}\right) b_{\mathbf{p}}^{{ }^{\dagger}} b_{\mathbf{p}}^{s}\right]
\end{aligned}
$$

where $m \neq \bar{m}$, i.e., the mass of a neutrino is not equal to the mass of the corresponding antineutrino. For $m \neq \bar{m}$ the new Hamiltonian violates CPT invariance and locality, since there is no possibility to derive it from any local QFT.

\section{CPT TESTS AT A NEUTRINO FAC- TORY}

As we have seen, CPT violation implies physics beyond local QFT, which means that we have to consider Planck scale physics, large extra dimensions, or string theory. In Ref. [6], CPT violation was used to accommodate the LSND result [15, 16, 17. . Here we will study precision measurements at a future neutrino factory, which will lead to interesting phenomenological limits on the differences in the neutrino-antineutrino masses and mixing angles.

Now, CPT invariance implies that

$$
\mathrm{P}\left(\nu_{\alpha} \rightarrow \nu_{\alpha^{\prime}}\right)=\mathrm{P}\left(\bar{\nu}_{\alpha^{\prime}} \rightarrow \bar{\nu}_{\alpha}\right),
$$

where $\mathrm{P}\left(\nu_{\alpha} \rightarrow \nu_{\alpha^{\prime}}\right)$ is the neutrino oscillation transition probability that $\nu_{\alpha} \rightarrow \nu_{\alpha^{\prime}}$ will occur. In the present discussion, we will violate CPT invariance and locality (but not necessarily Lorentz invariance) by assuming that masses and mixings differ for neutrinos and antineutrinos. For neutrinos we have $m_{a}$ and $U$, whereas for antineutrinos we have $\bar{m}_{a}$ and $\bar{U}$. Thus, as a consequence,

$$
\mathrm{P}\left(\nu_{\alpha} \rightarrow \nu_{\alpha^{\prime}}\right) \neq \mathrm{P}\left(\bar{\nu}_{\alpha^{\prime}} \rightarrow \bar{\nu}_{\alpha}\right),
$$

where for two neutrino flavors

$$
\begin{aligned}
\mathrm{P}\left(\nu_{\alpha} \rightarrow \nu_{\alpha^{\prime}}\right) & \equiv \delta_{\alpha \alpha^{\prime}} \\
& -\left(2 \delta_{\alpha \alpha^{\prime}}-1\right) \sin ^{2} 2 \theta \sin ^{2} \frac{\Delta m^{2} L}{4 E}, \\
\mathrm{P}\left(\bar{\nu}_{\alpha} \rightarrow \bar{\nu}_{\alpha^{\prime}}\right) & \equiv \delta_{\alpha \alpha^{\prime}} \\
& -\left(2 \delta_{\alpha \alpha^{\prime}}-1\right) \sin ^{2} 2 \bar{\theta} \sin ^{2} \frac{\Delta \bar{m}^{2} L}{4 E},
\end{aligned}
$$

which means that

$$
\begin{aligned}
\Delta \mathrm{P}_{\alpha \alpha}^{\mathrm{CPT}} & \equiv \mathrm{P}\left(\nu_{\alpha} \rightarrow \nu_{\alpha}\right)-\mathrm{P}\left(\bar{\nu}_{\alpha} \rightarrow \bar{\nu}_{\alpha}\right) \\
& =-\sin ^{2} 2 \theta \sin ^{2} \frac{\Delta m^{2} L}{4 E} \\
& -\sin ^{2} 2 \bar{\theta} \sin ^{2} \frac{\Delta \bar{m}^{2} L}{4 E} .
\end{aligned}
$$

For three neutrino flavors we have more complicated formulas. However, we will carry out our numerical calculations using three neutrino flavors.

At a neutrino factory, neutrinos would be produced in muon decays $\mu^{+} \rightarrow e^{+} \nu_{e} \bar{\nu}_{\mu}$ (or $\mu^{-} \rightarrow$ $\left.e^{-} \bar{\nu}_{e} \nu_{\mu}\right)$. The straightforward test of CPT violation would be to check the appearance relation $\mathrm{P}\left(\nu_{e} \rightarrow \nu_{\mu}\right)=\mathrm{P}\left(\bar{\nu}_{\mu} \rightarrow \bar{\nu}_{e}\right)\left[\right.$ or $\mathrm{P}\left(\bar{\nu}_{e} \rightarrow \bar{\nu}_{\mu}\right)=$ $\left.\mathrm{P}\left(\nu_{\mu} \rightarrow \nu_{e}\right)\right]$. However, this would require to measure the sign of the charge of the produced lepton, which could be hard. We propose instead to check the equality

$$
\mathrm{P}\left(\nu_{\mu} \rightarrow \nu_{\mu}\right)=\mathrm{P}\left(\bar{\nu}_{\mu} \rightarrow \bar{\nu}_{\mu}\right),
$$


i.e., the $\nu_{\mu}$ and $\bar{\nu}_{\mu}$ disappearance channels. The advantages of checking these disappearance channels are, for example:

- high event rates,

- no beam contamination, i.e., no relevant background is present,

- small matter effects,

- large neutrino oscillation effects.

Thus, we could obtain exclusion limits for tiny CPT-violating effects.

If we consider the $\nu_{\mu}$ and $\bar{\nu}_{\mu}$ channels as independent experiments, then CPT violation in neutrino oscillations can be quantified by the following asymmetries:

$$
\begin{aligned}
\delta & \equiv\left|\Delta m_{32}^{2}-\Delta \bar{m}_{32}^{2}\right|, \\
\epsilon & \equiv\left|\sin ^{2} 2 \theta_{23}-\sin ^{2} 2 \bar{\theta}_{23}\right| .
\end{aligned}
$$

For $m_{1} \ll \sqrt{\Delta m_{\odot}^{2}}$ (hierarchical) and $\left|m_{3}-\bar{m}_{3}\right| \ll$ $\left(m_{3}\right)_{\text {average }}$, we have [9]

$$
\begin{aligned}
\delta & \simeq 2 a_{\mathrm{CPT}} \Delta m_{32}^{2} \\
\epsilon & \simeq 2 b_{\mathrm{CPT}} \sqrt{\sin ^{2} 2 \theta_{23}} \sqrt{1-\sin ^{2} 2 \theta_{23}} \\
& \times \arcsin \sqrt{\sin ^{2} 2 \theta_{23}},
\end{aligned}
$$

where the asymmetry parameters are

$$
a_{\mathrm{CPT}} \equiv \frac{\left|m_{3}-\bar{m}_{3}\right|}{\left(m_{3}\right)_{\text {average }}}
$$

and

$$
b_{\mathrm{CPT}} \equiv \frac{\left|\theta_{23}-\bar{\theta}_{23}\right|}{\left(\theta_{23}\right)_{\text {average }}} .
$$

Next, we want to estimate the sensitivities $\delta a_{\mathrm{CPT}}$ and $\delta b_{\mathrm{CPT}}$ of the asymmetry parameters $a_{\mathrm{CPT}}$ and $b_{\mathrm{CPT}}$. The sensitivities to possible CPT violation are given by the accuracies with which $a_{\mathrm{CPT}}$ and $b_{\mathrm{CPT}}$ can be measured. Comparing $a_{\mathrm{CPT}}$ and $b_{\mathrm{CPT}}$ with the corresponding relative statistical errors $\delta \Delta m_{32}^{2}$ and $\delta \theta_{23}$ of the measurements of $\Delta m_{32}^{2}$ and $\theta_{23}$, we obtain the sensitivities of the asymmetry parameters 18. Thus, the sensitivities $\delta a_{\mathrm{CPT}}$ and $\delta b_{\mathrm{CPT}}$ for the asymmetry parameters $a_{\mathrm{CPT}}$ and $b_{\mathrm{CPT}}$ are given by:

$$
\begin{aligned}
\delta a_{\mathrm{CPT}} & \sim \frac{\delta \Delta m_{32}^{2}}{2}, \\
\delta b_{\mathrm{CPT}} & \sim \delta \theta_{23} .
\end{aligned}
$$

The result of a possible future neutrino factory setup simulation is presented in Fig. 1. For our

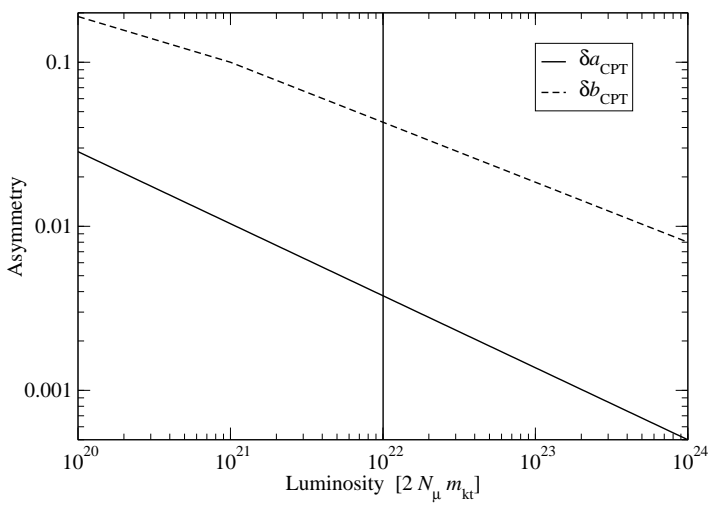

Figure 1. The sensitivities $\delta a_{\mathrm{CPT}}$ (solid curve) and $\delta b_{\mathrm{CPT}}$ (dashed curve) of an estimate of the asymmetries $a_{\mathrm{CPT}}$ and $b_{\mathrm{CPT}}$ as a function of the luminosity $\mathcal{L} \equiv 2 N_{\mu} m_{\mathrm{kt}}$. Parameter values: $E_{\nu}=50 \mathrm{GeV}$ (muon energy), $L=3000 \mathrm{~km}$ $\left(\Delta m_{23}^{2}\right.$, solid curve $) / L=7000 \mathrm{~km}\left(\theta_{23}\right.$, dashed curve), $m_{\mathrm{kt}}=10 \mathrm{kt}$ (mass of detector), $10^{20}$ muons/year, and 5 years (running time). The figure has been adopted from Ref. [9].

numerical calculations we assumed a $10 \mathrm{kt}$ detector and $10^{20}$ stored muons per year during 5 years. Using the obtained upper bounds in Fig. 1 $\left(a_{C P T} \lesssim 3.8 \cdot 10^{-3}\right.$ and $\left.b_{C P T} \lesssim 4.3 \cdot 10^{-2}\right)$ as well as $\left(m_{3}\right)_{\text {average }} \simeq \sqrt{\Delta m_{\text {atm }}^{2}} \leq 5 \cdot 10^{-2} \mathrm{eV}$ and $\left(\theta_{23}\right)_{\text {average }} \simeq \theta_{\mathrm{atm}}=45^{\circ}$, we find that

$$
\begin{aligned}
&\left|m_{3}-\bar{m}_{3}\right| \lesssim 1.9 \cdot 10^{-4} \mathrm{eV}, \\
&\left|\theta_{23}-\bar{\theta}_{23}\right| \lesssim 2^{\circ},
\end{aligned}
$$

which correspond to

$$
\begin{aligned}
a_{\mathrm{CPT}} & \lesssim 0.38 \%, \\
b_{\mathrm{CPT}} & \lesssim 4.3 \% .
\end{aligned}
$$

\section{SUMMARY \& CONCLUSIONS}

In summary, CPT violation is not allowed in local QFT. Thus, fundamental CPT violation 
would mean physics beyond local QFT and the SM, such as Planck scale physics, large extra dimensions, or string theory. Finally, we have shown in our neutrino factory setup simulation that CPT violation is detectable if $\left|m_{3}-\bar{m}_{3}\right| \not$ $1.9 \cdot 10^{-4} \mathrm{eV}$ and $\left|\theta_{23}-\bar{\theta}_{23}\right| \not 22^{\circ}$.

\section{Acknowledgments}

I would like to thank my co-workers Samoil M. Bilenky, Martin Freund, Manfred Lindner, and Walter Winter for fruitful collaboration and Håkan Snellman for proof-reading this proceeding.

This work was supported by the Swedish Foundation for International Cooperation in Research and Higher Education (STINT), the WennerGren Foundations, the Swedish Research Council (Vetenskapsrådet), Contract No. 621-2001-1611, and the Magnus Bergvall Foundation (Magn. Bergvalls Stiftelse).

\section{REFERENCES}

1. S.R. Coleman and S.L. Glashow, Phys. Lett. B405 (1997) 249, hep-ph/9703240.

2. S.R. Coleman and S.L. Glashow, Phys. Rev. D59 (1999) 116008, hep-ph/9812418.

3. V.D. Barger et al., Phys. Rev. Lett. 85 (2000) 5055, hep-ph/0005197.

4. H. Murayama and T. Yanagida, Phys. Lett. B520 (2001) 263, hep-ph/0010178.

5. M.C. Bañuls, G. Barenboim and J. Bernabeu, Phys. Lett. B513 (2001) 391, hep$\mathrm{ph} / 0102184$.

6. G. Barenboim et al., hep-ph/0108199.

7. Z.z. Xing, J. Phys. G28 (2002) B7, hep$\mathrm{ph} / 0112120$.

8. S. Skadhauge, Nucl. Phys. B639 (2002) 281, hep-ph/0112189.

9. S.M. Bilenky et al., Phys. Rev. D65 (2002) 073024, hep-ph/0112226.

10. G. Barenboim, L. Borissov and J. Lykken, Phys. Lett. B534 (2002) 106, hep-ph/0201080.

11. J.N. Bahcall, V. Barger and D. Marfatia, Phys. Lett. B534 (2002) 120, hep$\mathrm{ph} / 0201211$.
12. A. Strumia, Phys. Lett. B539 (2002) 91, hep$\mathrm{ph} / 0201134$.

13. I. Mocioiu and M. Pospelov, Phys. Lett. B534 (2002) 114, hep-ph/0202160.

14. G. Barenboim et al., Phys. Lett. B537 (2002) 227, hep-ph/0203261.

15. LSND Collaboration, C. Athanassopoulos et al., Phys. Rev. Lett. 77 (1996) 3082, nuclex/9605003.

16. LSND Collaboration, C. Athanassopoulos et al., Phys. Rev. Lett. 81 (1998) 1774, nuclex/9709006.

17. LSND Collaboration, A. Aguilar et al., Phys. Rev. D64 (2001) 112007, hep-ex/0104049.

18. M. Freund, P. Huber and M. Lindner, Nucl. Phys. B615 (2001) 331, hep-ph/0105071. 